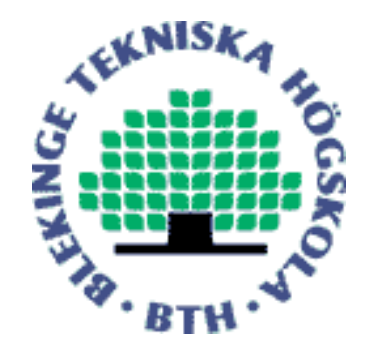

Copyright @ 2014 IEEE.

Citation for the published paper:

Title:

Two-Dimensional Spectrum for BiSAR Derivation Based on Lagrange Inversion Theorem

Author:

Viet Thuy Vu, Mats Pettersson, Thomas Sjögren

Journal:

GEOSCIENCE AND REMOTE SENSING LETTERS

Year:

2014

Vol:

11

Issue:

7

Pagination:

1210-1214

URL/DOI to the paper:

10.1109/LGRS.2013.2289735

This material is posted here with permission of the IEEE. Such permission of the IEEE does not in any way imply IEEE endorsement of any of BTH's products or services Internal or personal use of this material is permitted. However, permission to reprint/republish this material for advertising or promotional purposes or for creating new collective works for resale or redistribution must be obtained from the IEEE by sending a blank email message to pubs-permissions@iee.org.

By choosing to view this document, you agree to all provisions of the copyright laws protecting it. 


\title{
Two-Dimensional Spectrum for BiSAR Derivation Based on Lagrange Inversion Theorem
}

\author{
Viet T. Vu, Member, IEEE, Thomas K. Sjögren, Student Member, IEEE, \\ and Mats I. Pettersson, Member, IEEE
}

\begin{abstract}
A two-dimensional spectrum for bistatic synthetic aperture radar (BiSAR) is derived in this paper. The derivation is based on the commonly used mathematic principles such as the method of stationary phase and the Fourier transform and the Lagrange inversion theorem in order to find the point of stationary phase in the method of stationary phase. Using Lagrange inversion theorem allows minimizing the initial assumptions or the initial approximations. The derived twodimensional spectrum is compared to the commonly used twodimensional spectrum to verify it and illustrate its accuracy.
\end{abstract}

Index Terms-Bistatic SAR, two-dimensional spectrum, Lagrange inversion theorem, LORA.

\section{INTRODUCTION}

B ISTATIC SAR is used to indicate SAR systems whose transmitter and receiver(s) are separated. This geometry brings bistatic SAR systems some unique characteristics such as the flexibility in design, optimized vulnerability, 3D imaging capability and specially enhanced classification of targets. Reconstruction of SAR scenes from bistatic SAR echoes is based on algorithms which can be divided into two: time- and frequency-domain. The former refers to the backprojection algorithms [1], [2] where all calculations are implemented in time-domain. On the contrary, all processing steps of the latter such as Range Doppler (RD) [3], Chirp Scaling (CS) [4] are performed in one-dimension and/or twodimension frequency-domain. Since Fast Fourier Transform (FFT) is always utilized in the these algorithms, they are also called the FFT-based algorithms. Regardless of the limits of the frequency algorithms in e.g. signal bandwidth, pulse repetition frequency (PRF), integration time and so forth, the frequency algorithms are still good candidates for bistatic SAR processing thanks to its benefit of processing time.

Let's have a look at the bistatic SAR frequency-domain algorithms. It is very obvious that the frequency-domain algorithms in general are developed on a two-dimensional spectrum for bistatic SAR or in other words a two-dimensional spectrum for bistatic SAR is a key for the frequency-domain algorithm development. For this reason, the derivation of a two-dimensional spectrum for bistatic SAR is of great interest to the researchers in the field. A summary of different twodimensional spectra derived for bistatic SAR is given [5].

V. T. Vu, T. K. Sjögren and M. I. Pettersson are with the Department of Electrical Engineering, Blekinge Institute of Technology, Karlskrona, 37143 Sweden e-mail:viet.thuy.vu@bth.se.

Manuscript received February 20, 2012, revised June 20, 2012.
Among them, the two-dimensional spectrum for bistatic SAR processing based on series reversion [6] is shown to be the most accurate spectrum as it works well for different cases. The RD and CS algorithms [3], [4] are directly developed on this two-dimensional spectrum.

The aim of this study is to derive a new two-dimensional spectrum for bistatic SAR based on the commonly used mathematic principles such as the method of stationary phase and the Fourier transform. Point of stationary phase in the method of stationary phase is found by the Lagrange inversion theorem which is considered the crucial contribution of the study. Similar to the two-dimensional spectrum based on series reversion [6], the accuracy of the two-dimensional spectrum in the study can also be controlled by keeping enough terms in the series expansion. However, using the Lagrange inversion theorem instead of the series reversion method in [6] avoids the initial assumptions or the initial approximations.

The rest of the paper is organized as follows. Section II presents the bistatic signal model which will be used in the latter derivation. The derivation of the new two-dimensional spectrum for bistatic SAR based on Lagrange inversion theorem is introduced in section III. The introduced twodimensional spectrum is verified in section IV by a comparison between the new two-dimensional spectrum, the one based on series reversion and the ideal one. The comparison also illustrates the accuracy of the new two-dimensional spectrum. Section $\mathrm{V}$ provides the conclusions.

\section{Bistatic SAR Signal Model}

Let's consider a bistatic SAR system with the transmitter and the receiver separated in two different platforms. The bistatic SAR geometry is illustrated in Fig. 1 where the velocities of the platforms are arbitrary. The bistatic SAR system is assumed to utilize chirp pulses. After demodulation to baseband, radar echo which is reflected from a point-like scatterer in the illuminated ground scene is expressed by

$$
\begin{aligned}
s(t, \tau)= & w_{r}\left(\tau-\frac{R(t)}{\mathrm{c}}\right) w_{a z}(t) \\
& \exp \left\{-j 2 \pi f_{c} \frac{R(t)}{\mathrm{c}}+j \pi \kappa\left(\tau-\frac{R(t)}{\mathrm{c}}\right)^{2}\right\}
\end{aligned}
$$

where $t$ and $\tau$ are azimuth and range time, $w_{a z}(\cdot)$ and $w_{r}(\cdot)$ are the range and azimuth envelopes, $f_{c}$ is the center frequency, $\kappa$ is the chirp rate, and $R(t)$ is the range timedependent bistatic range - the two-way traveling distance of the chirp signal from the transmitter impinging on a target on 


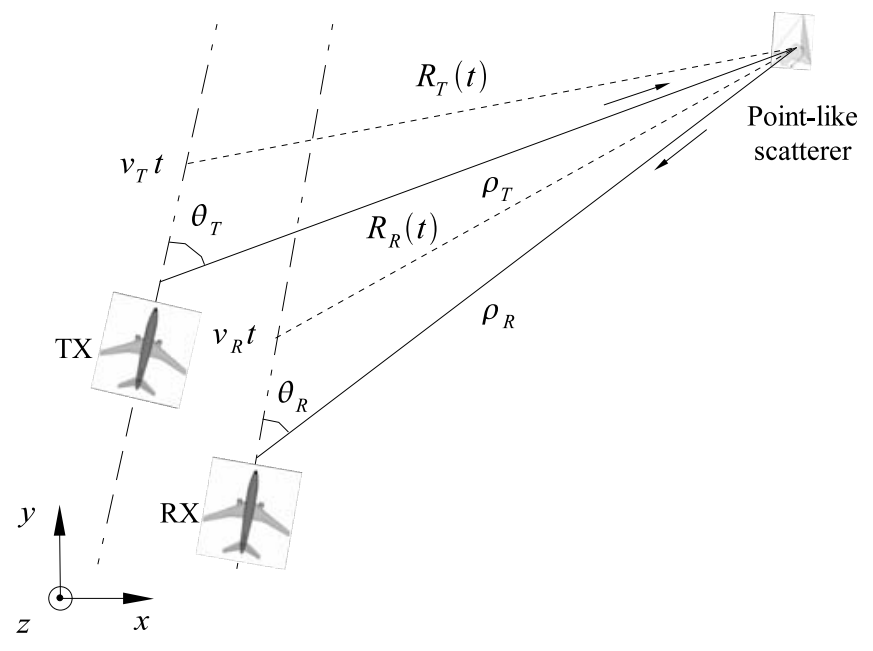

Fig. 1. Bistatic SAR geometry.

the SAR scene and scattered back to the receiver. With the bistatic SAR geometry given in Fig. 1, the bistatic range is calculated using the law of cosines as

$$
\begin{aligned}
R(t) & =R_{T}(t)+R_{R}(t) \\
& =\sqrt{v_{T}^{2} t^{2}-2 \rho_{T} \cos \theta_{T} v_{T} t+\rho_{T}^{2}} \\
& +\sqrt{v_{R}^{2} t^{2}-2 \rho_{R} \cos \theta_{R} v_{R} t+\rho_{R}^{2}}
\end{aligned}
$$

where the subscripts $T$ and $R$ imply the transmitter and the receiver, respectively. Hence, $v_{T}$ and $v_{R}$ are the speeds of the platforms, $\rho_{T}$ and $\rho_{R}$ are the ranges corresponding to the initial coordinates of the platform, i.e. $t=0, \theta_{T}$ and $\theta_{R}$ are the angles formed by $\left(\vec{v}_{T}, \vec{\rho}_{T}\right)$ and $\left(\vec{v}_{R}, \vec{\rho}_{R}\right)$, respectively. The Fourier transform in range of (1) using the method of stationary phase is similar to the one for monostatic case and can be easily proved to be

$$
\begin{aligned}
S\left(t, f_{\tau}\right)= & W_{r}\left(\frac{f_{\tau}}{\kappa}\right) w_{a z}(t) \\
& \exp \left\{-j 2 \pi\left(f_{c}+f_{\tau}\right) \frac{R(t)}{\mathrm{c}}-j \pi \frac{f_{\tau}^{2}}{\kappa}\right\}
\end{aligned}
$$

where $W_{r}(\cdot)$ represents the radar signal spectrum. However, the Fourier transform in azimuth of (3) to obtain a twodimensional Spectrum for bistatic SAR is more complicated in comparison to the monostatic case due to the double square root given by (2) in (3).

\section{Derivation of Two-Dimensional SPECtrum For BISTATIC SAR}

The two-dimensional spectrum for bistatic SAR is the Fourier transform in azimuth of (3) and is found from the Fourier integral

$$
S\left(f_{t}, f_{\tau}\right)=\int S\left(t, f_{\tau}\right) \exp \left\{-j 2 \pi f_{t} t\right\} d t
$$

Substituting (2) into (3) and then (3) into (4), the phase in the Fourier integral (4) will be given by

$$
\begin{aligned}
\phi\left(t, f_{\tau}\right)= & -\frac{2 \pi\left(f_{c}+f_{\tau}\right)}{\mathrm{c}} \sqrt{v_{T}^{2} t^{2}-2 \rho_{T} \cos \theta_{T} v_{T} t+\rho_{T}^{2}} \\
& -\frac{2 \pi\left(f_{c}+f_{\tau}\right)}{\mathrm{c}} \sqrt{v_{R}^{2} t^{2}-2 \rho_{R} \cos \theta_{R} v_{R} t+\rho_{R}^{2}} \\
& -\frac{\pi f_{\tau}^{2}}{\kappa}-2 \pi f_{t} t
\end{aligned}
$$

According to the method of stationary phase, the Fourier integral can be approximately estimated by

$$
S\left(f_{t}, f_{\tau}\right) \approx W_{r}\left(\frac{f_{\tau}}{\kappa}\right) W_{a z}(\tilde{t}) \exp \left\{j \phi\left(\tilde{t}, f_{\tau}\right)\right\}
$$

where $W_{a z}(\cdot)$ refers to the Doppler spectrum, $\tilde{t}$ is the point of stationary phase and the root(s) of the following equation

$$
\left.\frac{d}{d t} \phi\left(t, f_{\tau}\right)\right|_{t=\tilde{t}}=0
$$

Equation (7) can easily be rewritten as

$$
\begin{aligned}
f_{t}= & -\frac{f_{c}+f_{\tau}}{\mathrm{c}} \frac{v_{T}^{2} \tilde{t}-\rho_{T} \cos \theta_{T} v_{T}}{\left(v_{T}^{2} \tilde{t}^{2}-2 \rho_{T} \cos \theta_{T} v_{T} \tilde{t}+\rho_{T}^{2}\right)^{1 / 2}} \\
& -\frac{f_{c}+f_{\tau}}{\mathrm{c}} \frac{v_{R}^{2} \tilde{t}-\rho_{R} \cos \theta_{R} v_{R}}{\left(v_{R}^{2} \tilde{t}^{2}-2 \rho_{R} \cos \theta_{R} v_{R} \tilde{t}+\rho_{R}^{2}\right)^{1 / 2}}
\end{aligned}
$$

Solving (8) to find the expression of the point of stationary phase $\tilde{t}$ requires much effort. However, we can use the Lagrange inversion theorem to find approximately solutions for $\tilde{t}$. As we see in (8), $f_{t}$ is a function of $\tilde{t}$, i.e. $f_{t}=h(\tilde{t})$. According to the Lagrange inversion theorem, if $h$ is analytic at a point $\tilde{t}_{0}$ and its first derivative $h^{\prime}\left(\tilde{t}_{0}\right) \neq 0$, then it is possible to invert or solve the equation for $\tilde{t}$, i.e. $\tilde{t}=g\left(f_{t}\right)$ on a neighborhood of $\tilde{t}_{0}$, where $g$ is analytic at the point $h\left(\tilde{t}_{0}\right)$. The series expansion of $g$ is given by

$g\left(f_{t}\right)=\tilde{t}_{0}+\sum_{n=1}^{\infty} \lim _{\tilde{t} \rightarrow \tilde{t}_{0}} \frac{\left[f_{t}-h\left(\tilde{t}_{0}\right)\right]^{n}}{n !} \frac{d^{n-1}}{d t^{n-1}}\left[\frac{\tilde{t}-\tilde{t}_{0}}{h(\tilde{t})-h\left(\tilde{t}_{0}\right)}\right]^{n}$

It is obvious that there are different ways to find the function $g\left(f_{t}\right)$ based on the selection of $\tilde{t}_{0}$. In this study, we present only one solution for $g\left(f_{t}\right)$. Let's consider the simple value $\tilde{t}_{0}=0$. For relatively short apertures, the function $h(\tilde{t})$ is analytic at the point $\tilde{t}_{0}=0$ since $R(t)$ calculated by (2) can be locally given by a convergent power series [6]. The first derivative of $h(\tilde{t})$ is expressed by

$$
\begin{aligned}
\frac{d}{d t} h(\tilde{t}) & =-\frac{f_{c}+f_{\tau}}{\mathrm{c}} \frac{\rho_{T}^{2} \sin ^{2} \theta_{T} v_{T}^{2}}{\left(v_{T}^{2} \tilde{t}^{2}-2 \rho_{T} \cos \theta_{T} v_{T} \tilde{t}+\rho_{T}^{2}\right)^{3 / 2}} \\
& =-\frac{f_{c}+f_{\tau}}{\mathrm{c}} \frac{\rho_{R}^{2} \sin ^{2} \theta_{R} v_{R}^{2}}{\left(v_{R}^{2} \tilde{t}^{2}-2 \rho_{R} \cos \theta_{R} v_{R} \tilde{t}+\rho_{R}^{2}\right)^{3 / 2}}
\end{aligned}
$$

and the value of the first derivative of $h$ at the point $\tilde{t}=0$

$$
\frac{d}{d t} h(\tilde{t}=0)=-\frac{f_{c}+f_{\tau}}{\mathrm{c}}\left(\frac{\sin ^{2} \theta_{T} v_{T}^{2}}{\rho_{T}}+\frac{\sin ^{2} \theta_{R} v_{R}^{2}}{\rho_{R}}\right) \neq 0
$$


It is therefore possible to solve the equation for $\tilde{t}=g\left(f_{t}\right)$ on a neighborhood of $\tilde{t}_{0}=0$ and the series expansion of $g$ is reduced to

$$
g\left(f_{t}\right)=\sum_{n=1}^{\infty} \lim _{\tilde{t} \rightarrow 0} \frac{\left[f_{t}-h(0)\right]^{n}}{n !} \frac{d^{n-1}}{d t^{n-1}}\left[\frac{\tilde{t}}{h(\tilde{t})-h(0)}\right]^{n}
$$

where

$$
h(0)=-\frac{f_{c}+f_{\tau}}{\mathrm{c}}\left(-\cos \theta_{T} v_{T}-\cos \theta_{R} v_{R}\right)=-\frac{f_{c}+f_{\tau}}{\mathrm{c}} k_{0}
$$

Since the point of stationary phase $\tilde{t}=g\left(f_{t}\right)$ is a power series, its accuracy is controlled by the number of terms. For example, if we consider the terms in (12) up to the third-order, the point of stationary phase will be given by

$$
\begin{aligned}
\tilde{t} & =\frac{\left[f_{t}-h(0)\right]^{1}}{1 !} \lim _{\tilde{t} \rightarrow 0}\left[\frac{\tilde{t}}{h(\tilde{t})-h(0)}\right] \\
& +\frac{\left[f_{t}-h(0)\right]^{2}}{2 !} \lim _{\tilde{t} \rightarrow 0} \frac{d^{1}}{d \tilde{t}^{1}}\left[\frac{\tilde{t}}{h(\tilde{t})-h(0)}\right]^{2} \\
& +\frac{\left[f_{t}-h(0)\right]^{3}}{3 !} \lim _{\tilde{t} \rightarrow 0} \frac{d^{2}}{d \tilde{t}^{2}}\left[\frac{\tilde{t}}{h(\tilde{t})-h(0)}\right]^{3}
\end{aligned}
$$

The finding the limits in (13) are presented in Appendix A. The final expression for the point of stationary phase up to the third-order is given by

$$
\begin{aligned}
\tilde{t}= & -\frac{1}{k_{1}}\left(\frac{\mathrm{c}}{f_{c}+f_{\tau}} f_{t}+k_{0}\right)-\frac{k_{2}}{k_{1}}\left[\frac{1}{k_{1}}\left(\frac{\mathrm{c}}{f_{c}+f_{\tau}} f_{t}+k_{0}\right)\right]^{2} \\
& -\left(\frac{2 k_{2}^{2}}{k_{1}^{2}}-\frac{k_{3}}{2 k_{1}}\right)\left[\frac{1}{k_{1}}\left(\frac{\mathrm{c}}{f_{c}+f_{\tau}} f_{t}+k_{0}\right)\right]^{3}
\end{aligned}
$$

where

$$
\begin{gathered}
k_{1}=\frac{\sin ^{2} \theta_{T} v_{T}^{2}}{\rho_{T}}+\frac{\sin ^{2} \theta_{R} v_{R}^{2}}{\rho_{R}} \\
k_{2}=\frac{3 \sin ^{2} \theta_{T} \cos \theta_{T} v_{T}^{3}}{\rho_{T}^{2}}+\frac{3 \sin ^{2} \theta_{R} \cos \theta_{R} v_{R}^{3}}{\rho_{R}^{2}}
\end{gathered}
$$

and

$$
\begin{aligned}
k_{3} & =\frac{3 \sin ^{2} \theta_{T}\left(4 \cos ^{2} \theta_{T}-\sin ^{2} \theta_{T}\right) v_{T}^{4}}{\rho_{T}^{3}} \\
& +\frac{3 \sin ^{2} \theta_{R}\left(4 \cos ^{2} \theta_{R}-\sin ^{2} \theta_{R}\right) v_{R}^{4}}{\rho_{R}^{3}}
\end{aligned}
$$

The simulation results presented in the next section shows that this series (12) is useful with the consideration up to the thirdorder. Substituting the point of stationary given by (15) into (6) results in the two-dimensional spectrum for bistatic SAR.

\section{Simulation Results}

To verify and illustrate the accuracy of the two-dimensional spectrum for bistatic SAR introduced in the previous section, it is common knowledge to carry out these tasks in the following steps. Firstly, a bistatic SAR data set of a single pointlike scatterer is simulated in the time-domain. Secondly, the data set is transformed to two-dimensional frequency-domain. Thirdly, the matched filtering for the data set is performed in
TABLE I

PARAMETERS FOR SIMULATIONS.

\begin{tabular}{ccc}
\hline \hline Parameter & Transmitter & Receiver \\
\hline Operating frequency range & $307 \mathrm{MHz}-333 \mathrm{MHz}$ & \\
Platform speed & $130 \mathrm{~m} / \mathrm{s}$ & $125 \mathrm{~m} / \mathrm{s}$ \\
Flight altitude & $3000 \mathrm{~m}$ & $5000 \mathrm{~m}$ \\
PRF & $1300 \mathrm{~Hz}$ & \\
Aperture step & $0.1 \mathrm{~m}$ & \\
Number of aperture positions & $6001 / 15001$ & \\
Minimum range & $6000 \mathrm{~m}$ & \\
Direction of movement & $5^{\mathrm{O}}$ & $45^{\mathrm{O}}$ \\
\hline \hline
\end{tabular}

two-dimensional frequency-domain using the proposed twodimensional spectrum for bistatic SAR based on Lagrange inversion theorem and the ideal two-dimensional spectrum which can be obtained with the conjugate complex of the data set. The matched filtering can also performed using other two-dimensional spectra, e.g. the two-dimensional spectrum for bistatic SAR based on series reversion derived in [6] which is known as the most accurate two-dimensional spectrum for bistatic SAR. This helps to give a better evaluation for the two-dimensional spectrum for bistatic SAR introduced in this study. Fourthly, the matched filtering data sets are transformed back to time-domain. Finally, the differently focused results of the point-like scatterer are examined.

As indicated in the simulation results in [5], the focusing is strongly affected by the integration time. It is necessary to take this issue into account in the verification and illustration of the accuracy of the introduced two-dimensional spectrum. Hence, we need only to use a single set of parameters to simulate a bistatic SAR system and change the integration time of that simulated SAR system. The increase in integration time is nothing else than the increase in aperture length or in other words the increase in number of aperture positions. However, the increase in number of aperture positions is limited since this may violate the analytic characteristic of $h(\tilde{t})$ at $\tilde{t}=0$. In the following simulations, the LORA's parameter set configured for ground moving target indication (GMTI) purposes [7] is used for the transmitter while the motion parameters of CARABAS-II for the receiver [8]. Table I provides this set of parameters. There are three values for number of aperture positions to be used: 6001 - the original value - and 15001 - the modified values. They correspond to Case I and Case II. The platforms' direction of movements are arbitrarily selected by $5^{\circ}$ for the transmitter and $45^{\circ}$ for the receiver.

\section{A. Case I}

For Case I, the aperture length is $6000 \times 0.1 \mathrm{~m}=600 \mathrm{~m}$. The ratio of aperture length to the minimum range with respect to the transmitter is $600 \mathrm{~m} / 6000 \mathrm{~m}=0.1$. A similar ratio can be obtained with respect to the receiver. Such the value is small enough to ensure that the function $h(\tilde{t})$ is analytic at the point $\tilde{t}_{0}=0$. Fig. 2 shows the focusing results of the pointlike scatterer using two-dimensional spectrum for bistatic SAR based on Lagrange inversion theorem and the one based 
(a) Ideal

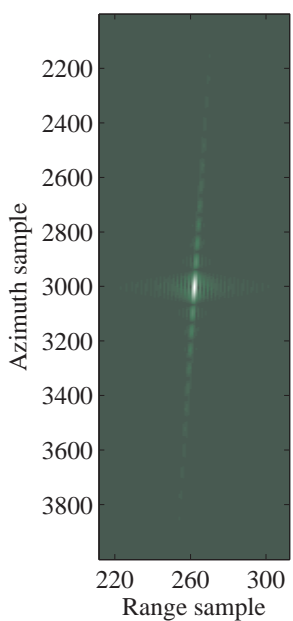

(b) Lagrange

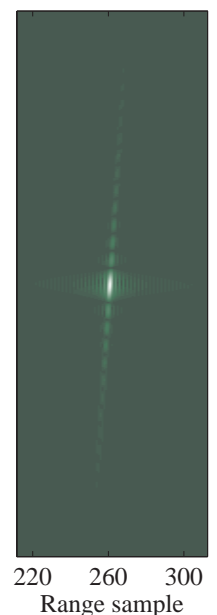

(c) Series

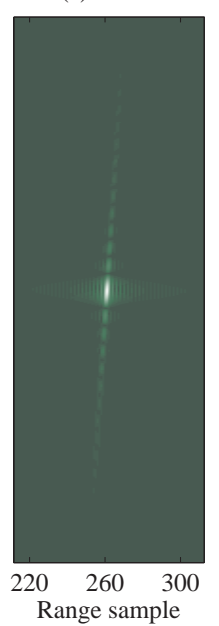

Fig. 2. Focusing results of the point-like scatterer in Case I using (a) ideal two-dimensional spectrum. (b) two-dimensional spectrum for bistatic SAR based on Lagrange inversion theorem. (c) two-dimensional spectrum for bistatic SAR based on series reversion.

on series reversion for matched filtering in two-dimensional frequency-domain. The focusing result using the ideal twodimensional spectrum of the point-like scatterer using twodimensional spectrum for bistatic SAR based on Lagrange inversion theorem and the one based on series reversion for matched filtering in two-dimensional frequency-domain. To observe the focusing results easily, we show only the center parts of the processed signal matrices. As shown in Fig. 2, there is almost no different between the focusing results using three different spectra for matched filtering. This verifies the two-dimensional spectrum for bistatic SAR based on Lagrange inversion theorem introduced in this study.

\section{B. Case II}

For Case II, the aperture length is increased up to $15000 \times$ $0.1 \mathrm{~m}=1500 \mathrm{~m}$. The ratio of aperture length to the minimum range with respect to the transmitter therefore increases to $1500 \mathrm{~m} / 6000 \mathrm{~m}=0.25$. Such the value is quite large but still sufficient to ensure that the function $h(\tilde{t})$ is analytic at the point $\tilde{t}_{0}=0$. The focusing results of the point-like scatterer using different two-dimensional spectrum are given in Fig. 3. These results indicate that both the two-dimensional spectrum based on Lagrange inversion theorem and the one based on series reversion for matched filtering in two-dimensional frequency-domain still work. However, as seen in Fig. 3, the performance of the former is little better than the later. Hence, the small defocusing can be observed from Fig. 3.c while the focusing results given in Fig. 3.a and Fig. 3.b are still very similar. This can be explain by the approximations for $R(t)$ in the derivation of the two-dimensional spectrum based on series reversion [6]. To get a better evaluation, we can extract the azimuth vectors from Fig. 3.a, Fig. 3.b and Fig. 3.c and plot them in a the same two-dimensional graph. This two-dimensional graph is shown in Fig. 4 and it clearly illustrates the performance of the two-dimensional spectrum based on Lagrange inversion theorem introduced in this study

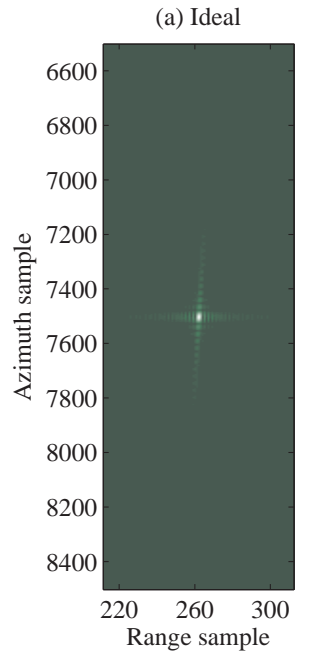

(b) Lagrange

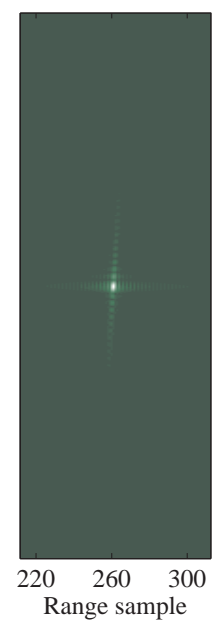

(c) Series

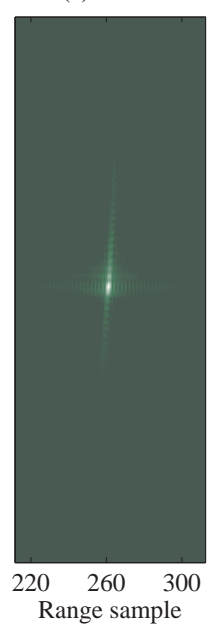

Fig. 3. Focusing results of the point-like scatterer in Case II using (a) ideal two-dimensional spectrum. (b) two-dimensional spectrum for bistatic SAR based on Lagrange inversion theorem. (c) two-dimensional spectrum for bistatic SAR based on series reversion.

in comparison to the ideal spectrum and the two-dimensional spectrum based on series reversion.

\section{CONCLUSION}

A new two-dimensional spectrum for bistatic SAR based on Lagrange inversion theorem is introduced in this study. Beside the commonly used mathematic principles such as the method of stationary phase and the Fourier transform, the spectrum is derived with the help of Lagrange inversion theorem. This allows avoiding the initial approximations which are ussually used in the derivation of other spectra. The proposed spectrum is only one of possibly different spectra which can be derived based on Lagrange inversion theorem. With a comparison of the proposed spectrum to others - the ideal spectrum and the spectrum based on series reversion, the proposed spectrum has been verified and its accuracy has been illustrated.

\section{APPENDIX A}

Let's consider the first limit of (14) where the limit of the numerator

$$
\lim _{\tilde{t} \rightarrow 0} \tilde{t}=0
$$

and the limit of the denominator

$$
\lim _{\tilde{t} \rightarrow 0} h(\tilde{t})-h(0)=0
$$

Hence, the first limit in (14) has the form $0 / 0$. One possibility to find such the limits is to applied L'Hopital's rule. Thus, L'Hopital's rule states that

$$
\begin{aligned}
\lim _{\tilde{t} \rightarrow 0} & {\left[\frac{\tilde{t}}{h(\tilde{t})-h(0)}\right]=\lim _{\tilde{t} \rightarrow 0}\left[\frac{(\tilde{t})^{\prime}}{(h(\tilde{t})-h(0))^{\prime}}\right] } \\
& =\lim _{\tilde{t} \rightarrow 0}\left[\frac{1}{(h(\tilde{t}))^{\prime}}\right] \\
& =\left[-\frac{f_{c}+f_{\tau}}{\mathrm{c}}\left(\frac{\sin ^{2} \theta_{T} v_{T}^{2}}{\rho_{T}}+\frac{\sin ^{2} \theta_{R} v_{R}^{2}}{\rho_{R}}\right)\right]^{-1}
\end{aligned}
$$


(a) Ideal

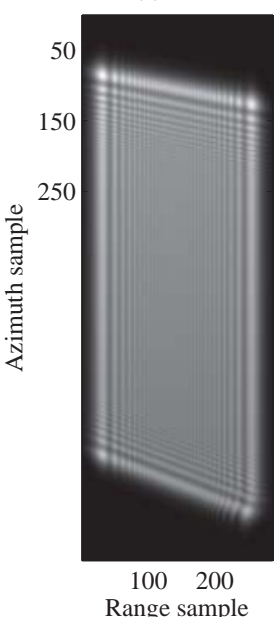

(b) Lagrange

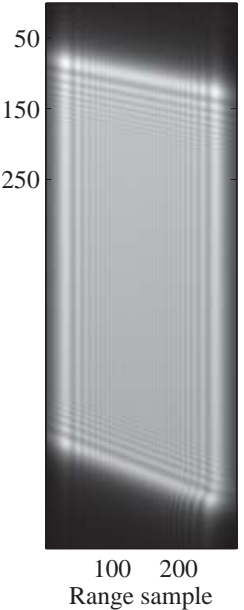

(c) Series

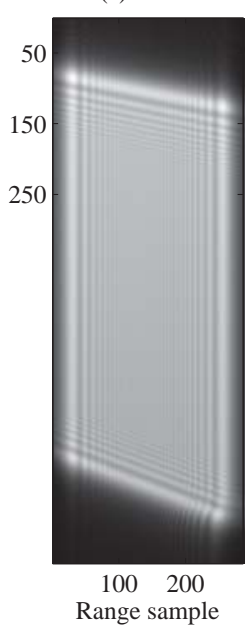

Fig. 4. Focusing results of the point-like scatterer in Case II shown by the the graph of the azimuth vectors extracted from Fig. 3.a, 3.b and 3.c.

where

$$
\begin{aligned}
\frac{d^{1}}{d \tilde{t}^{1}} h(\tilde{t})= & -\frac{f_{c}+f_{\tau}}{\mathrm{c}} \frac{\rho_{T}^{2} \sin ^{2} \theta_{T} v_{T}^{2}}{\left(v_{T}^{2} \tilde{t}^{2}-2 \rho_{T} \cos \theta_{T} v_{T} \tilde{t}+\rho_{T}^{2}\right)^{3 / 2}} \\
& -\frac{f_{c}+f_{\tau}}{\mathrm{c}} \frac{\rho_{R}^{2} \sin ^{2} \theta_{R} v_{R}^{2}}{\left(v_{R}^{2} \tilde{t}^{2}-2 \rho_{R} \cos \theta_{R} v_{R} \tilde{t}+\rho_{R}^{2}\right)^{3 / 2}}(
\end{aligned}
$$

The second limit in (14) can also be proved to have the form $0 / 0$. However, we need first to calculate the derivative of the function inside the limit

$$
\begin{aligned}
\lim _{\tilde{t} \rightarrow 0} & \frac{d^{1}}{d \tilde{t}^{1}}\left[\frac{\tilde{t}}{h(\tilde{t})-h(0)}\right]^{2}= \\
& 2 \lim _{\tilde{t} \rightarrow 0}\left[\frac{\tilde{t}}{h(\tilde{t})-h(0)}\right]\left[\frac{h(\tilde{t})-h(0)-\tilde{t}(h(\tilde{t}))^{\prime}}{(h(\tilde{t})-h(0))^{2}}\right] \text { (A-5) }
\end{aligned}
$$

Based on the algebraic limit theorem, the limit (A-5) can be separated into sums and products of more simple limits as

$$
\begin{aligned}
\lim _{\tilde{t} \rightarrow 0} & \frac{d^{1}}{d \tilde{t}^{1}}\left[\frac{\tilde{t}}{h(\tilde{t})-h(0)}\right]^{2} \\
& =2 \lim _{\tilde{t} \rightarrow 0}\left[\frac{\tilde{t}}{h(\tilde{t})-h(0)}\right] \lim _{\tilde{t} \rightarrow 0}\left[\frac{1}{h(\tilde{t})-h(0)}\right] \\
& -2 \lim _{\tilde{t} \rightarrow 0}\left[\frac{\tilde{t}}{h(\tilde{t})-h(0)}\right] \lim _{\tilde{t} \rightarrow 0}\left[\frac{\tilde{t}(h(\tilde{t}))^{\prime}}{(h(\tilde{t})-h(0))^{2}}\right] \\
& =2 \lim _{\tilde{t} \rightarrow 0}\left[\frac{\tilde{t}}{h(\tilde{t})-h(0)}\right] \lim _{\tilde{t} \rightarrow 0}\left[\frac{1}{h(\tilde{t})-h(0)}\right] \\
& -2\left\{\lim _{\tilde{t} \rightarrow 0}\left[\frac{\tilde{t}}{h(\tilde{t})-h(0)}\right]\right\} \lim _{\tilde{t} \rightarrow 0}^{2}\left[\frac{(h(\tilde{t}))^{\prime}}{h(\tilde{t})-h(0)}\right]
\end{aligned}
$$

Applying separately L'Hopital's rule to the limits in (A-6) results in

$$
\begin{aligned}
& \lim _{\tilde{t} \rightarrow 0} \frac{d^{1}}{d \tilde{t}^{1}}\left[\frac{\tilde{t}}{h(\tilde{t})-h(0)}\right]^{2} \\
&=2 \lim _{\tilde{t} \rightarrow 0}\left[\frac{1}{(h(\tilde{t}))^{\prime}}\right] \lim _{\tilde{t} \rightarrow 0}\left[\frac{0}{(h(\tilde{t}))^{\prime}}\right] \\
&-2\left\{\lim _{\tilde{t} \rightarrow 0}\left[\frac{1}{(h(\tilde{t}))^{\prime}}\right]\right\}^{2} \lim _{\tilde{t} \rightarrow 0}\left[\frac{(h(\tilde{t}))^{\prime \prime}}{(h(\tilde{t}))^{\prime}}\right] \\
&=-2\left(\frac{3 \sin ^{2} \theta_{T} \cos \theta_{T} v_{T}^{3}}{\rho_{T}^{2}}+\frac{3 \sin ^{2} \theta_{R} \cos \theta_{R} v_{R}^{3}}{\rho_{R}^{2}}\right) \\
& \times\left(-\frac{f_{c}+f_{\tau}}{\mathrm{c}}\right)^{-2}\left(\frac{\sin ^{2} \theta_{T} v_{T}^{2}}{\rho_{T}}+\frac{\sin ^{2} \theta_{R} v_{R}^{2}}{\rho_{R}}\right)^{-3}
\end{aligned}
$$

where

$$
\begin{aligned}
\frac{d^{2}}{d \tilde{t}^{2}} h(\tilde{t})= & -\frac{f_{c}+f_{\tau}}{\mathrm{c}} \frac{3 \rho_{T}^{2} \sin ^{2} \theta_{T} v_{T}^{2}\left(v_{T}^{2} t-\rho_{T} \cos \theta_{T} v_{T}\right)}{\left(v_{T}^{2} \tilde{t}^{2}-2 \rho_{T} \cos \theta_{T} v_{T} \tilde{t}+\rho_{T}^{2}\right)^{5 / 2}} \\
& -\frac{f_{c}+f_{\tau}}{\mathrm{c}} \frac{3 \rho_{R}^{2} \sin ^{2} \theta_{R} v_{R}^{2}\left(v_{R}^{2} t-\rho_{R} \cos \theta_{R} v_{R}\right)}{\left(v_{R}^{2} \tilde{t}^{2}-2 \rho_{R} \cos \theta_{R} v_{R} \tilde{t}+\rho_{R}^{2}\right)^{5 / 2}}(\mathrm{~A}-8)
\end{aligned}
$$

The third limit in (14) has also the form $0 / 0$. We can also apply L'Hopital's rule to find this limit after taking derivative of the functions inside the limits and splitting them into sums and products of more simple limits. The final expression of the third limit in (14) is given by

$$
\begin{aligned}
\lim _{\tilde{t} \rightarrow 0} \frac{d^{2}}{d \tilde{t}^{2}}\left[\frac{\tilde{t}}{h(\tilde{t})-h(0)}\right]^{3} \\
=12\left(\frac{3 \sin ^{2} \theta_{T} \cos \theta_{T} v_{T}^{3}}{\rho_{T}^{2}}+\frac{3 \sin ^{2} \theta_{R} \cos \theta_{R} v_{R}^{3}}{\rho_{R}^{2}}\right)^{2} \\
\times\left(-\frac{f_{c}+f_{\tau}}{\mathrm{c}}\right)^{-3}\left(\frac{\sin ^{2} \theta_{T} v_{T}^{2}}{\rho_{T}}+\frac{\sin ^{2} \theta_{R} v_{R}^{2}}{\rho_{R}}\right)^{-5} \\
-3\left(\frac{3 \sin ^{2} \theta_{T}\left(4 \cos ^{2} \theta_{T}-\sin ^{2} \theta_{T}\right) v_{T}^{4}}{\rho_{T}^{3}}\right)^{-3} \rho_{R}^{3}\left(\frac{\sin ^{2} \theta_{T} v_{T}^{2}}{\rho_{T}}+\frac{\sin ^{2} \theta_{R} v_{R}^{2}}{\rho_{R}}\right)^{-4}(\mathrm{~A}-9)
\end{aligned}
$$

\section{ACKNOWLEDGMENT}

The authors would like to thank the KK-Foundation for making this research project possible, and are also appreciative of support from the Swedish Defence Research Agency, Saab Bofors Dynamics, Saab Microwave Systems and RUAG Space.

\section{REFERENCES}

[1] V. T. Vu, T. K. Sjögren, and M. I. Pettersson, "Phase error calculation for fast time-domain bistatic SAR algorithms," IEEE Trans. Aerosp. Electron. Syst., vol. 48, no. 1, pp. 38-46, 2013. 
[2] V. T. Vu, T. K. Sjögren, and M. I. Pettersson, "Fast time-domain algorithms for UWB bistatic SAR processing," IEEE Trans. Aerosp. Electron. Syst., second revision for publication.

[3] Y. L. Neo, F. H. Wong, and I. G. Cumming, "Processing of azimuthinvariant bistatic SAR data using the range Doppler algorithm," IEEE Trans. Geosci. Remote Sensing, , vol. 46, no. 1, pp. 14-21, 2008.

[4] F. H. Wong, Y. L. Neo, and I. G. Cumming, "Focusing bistatic SAR data using the nonlinear chirp scaling algorithm," IEEE Trans. Geosci. Remote Sensing, , vol. 46, no. 9, pp. 2493-2505, 2008.

[5] K. Yang, F. He, and D. Liang, "A two-dimensional spectrum for general bistatic SAR processing," IEEE Geosci. Remote Sens. Lett., vol. 4, no. 1, pp. 108-112, 2010.

[6] Y. L. Neo, F. H. Wong, and I. G. Cumming, "A two-dimensional spectrum for bistatic SAR processing using series reversion," IEEE Geosci. Remote Sens. Lett., vol. 4, no. 1, pp. 93-96, 2007.

[7] L. M. H. Ulander and H. Hellsten, "Low-frequency ultrawideband arrayantenna SAR for stationary and moving target imaging," in Proc. SPIE Radar Sensor Technology IV, vol. 3704, Orlando, FL, Apr. 1999, pp. $149-158$.

[8] A. Gustavsson, L. M. H. Ulander, B. H. Flood, P.-O. Frölind, H. Hellsten, T. Jonsson, B. Larsson, and G. Stenstrom, "Development and operation of an airborne VHF SAR system-lessons learned," in Proc. IEEE IGARSS'98, vol. 1, Seattle, WA, Jul. 1998, pp. 458-462. 